\title{
Platelet adhesion on commercially pure titanium plates in vitro l: effects of plasma components and involvement of the von Willebrand factor and fibronectin
}

\author{
Akira Takahashi ${ }^{1}$, Shotaro Takahashi ${ }^{1}$, Tetsuhiro Tsujino ${ }^{2}$, Kazushige Isobe ${ }^{2}$, Taisuke Watanabe ${ }^{3}$, Yutaka Kitamura ${ }^{4}$,
} Takao Watanabe ${ }^{5}$, Koh Nakata ${ }^{6}$ and Tomoyuki Kawase ${ }^{7 *}$ (D)

\begin{abstract}
Background: Platelet-rich plasma (PRP) is widely used in regenerative dentistry. Furthermore, it is often applied in the pretreatment of titanium implants to improve their surface bioaffinity and initial stability. However, effects of PRP application on implant surface at cellular and molecular levels remain poorly understood. Therefore, we examined platelet adhesion on commercially pure titanium ( $c p$-Ti) plates, with a particular focus on fibrinogen (FGN), von Willebrand factor (VWF), and fibronectin (FN), in the presence or absence of plasma components.

Methods: Citrated blood samples were obtained from six healthy male volunteers, and pure-PRP (P-PRP) and pure platelet suspensions in phosphate-buffered saline (PBS) were prepared. Platelet adhesion on $c p$-Ti plate surface was evaluated by phalloidin staining and tetrazolium dye assay. Distribution of FGN, VWF, FN, albumin, CD62P, and CD63 was examined by immunocytochemical analysis.

Results: Platelets in PBS suspensions rapidly and time-dependently adhered to $c p$-Ti plate surface, but this adhesion was substantially disturbed by the presence of plasma components. FGN was most preferably adsorbed regardless of the presence or absence of plasma components, while VWF and FN showed greater accumulation on platelet adhesion area.

Conclusions: Although FGN is rapidly and abundantly adsorbed on cp-Ti plate surface, vWF and FN function as major platelet adhesion molecules in citrated blood samples. After pretreatment with P-PRP, however, platelets adhered to $c p$-Ti much less efficiently. Therefore, P-PRP pretreatment might not directly contribute to surface functionalization, initial stabilization, and osseointegration of machined or similar types of implants.
\end{abstract}

Keywords: Platelet, Titanium, Adhesion, Fibronectin, von Willebrand factor, Fibrinogen

\section{Introduction}

Platelets rapidly accumulate at the sites of injury to prevent bleeding and repair damaged tissue and organs by releasing growth factors [1]. This fundamental function of platelets is the basis of regenerative therapy using platelet-rich plasma (PRP) [2]. However, platelets in PRP are considered mainly as growth factor carriers. To maximize the potential of PRP and expand its clinical

\footnotetext{
* Correspondence: kawase@dent.niigata-u.ac.jp

${ }^{7}$ Division of Oral Bioengineering, Institute of Medicine and Dentistry, Niigata

University, Niigata, Japan

Full list of author information is available at the end of the article
}

applicability, clinicians must precisely understand functions of platelets in the form of PRP as well as analyze the quality of individual PRP preparations prior to clinical use.

PRP is often used in regenerative dentistry to acclimatize dental implant surface to in vivo conditions and, more specifically, to functionalize the surface to improve its bioaffinity [3-10]. The ultimate goal of this challenging application is to enhance the osseointegration of dental implants. Although upon implantation, dental implants probably immediately trigger coagulation and activate inflammatory reactions in vivo, there is no evidence regarding the mutual interactions between dental implants 
and citrated PRP in vitro. Additionally, the stability of PRP-coated implants in vivo remains unknown. Furthermore, the extent of platelet adhesion and adsorption of platelet adhesion molecules, along with platelet-derived growth factors and other plasma components, on implants surface remain unclear. To our knowledge, the use of PRP is not supported by scientific evidence to date, and its effect on implant stabilization and osseointegration remains controversial.

Given this, in this study, we established an experimental system using plain commercially pure titanium $(c p-\mathrm{Ti})$ plates to examine platelet adhesion and adsorption of platelet adhesion molecules [fibrinogen (FGN), von Willebrand factor (vWF), and fibronectin (FN)] on implant surface in the presence and absence of plasma components. FGN was specifically and abundantly adsorbed, but vWF and FN were the major platelet adhesion molecules on $c p$-Ti plate surface both in the presence and absence of plasma components.

\section{Materials and methods \\ Preparation of platelet suspensions in phosphate- buffered saline and plasma}

Blood samples were collected from six non-smoking healthy male volunteers aged 23 to 58 years. The study design and consent forms for all procedures were approved by the ethics committee for human participants at the Niigata University School of Medicine (Niigata, Japan) and complied with the Helsinki Declaration of 1964, as revised in 2013.

First, $\sim 9 \mathrm{~mL}$ of peripheral blood was collected in plain plastic vacuum blood collection tubes (Neotube; NIPRO, Osaka, Japan) containing $1 \mathrm{~mL}$ of $\mathrm{A}$ formulation of acid-citrate-dextrose (ACD-A; Terumo, Tokyo, Japan) $[11,12]$. Whole-blood samples were stored in a rotating agitator at ambient temperature and were used within $48 \mathrm{~h}[13,14]$. Thereafter, the samples were centrifuged at $472 \times g$ for $10 \mathrm{~min}$ (soft spin). The upper plasma fraction, $\sim 2 \mathrm{~mm}$ beyond the interface between the plasma and red blood cell fractions, was then transferred into 2-mL sample tubes, incubated with $5 \mu \mathrm{g} / \mathrm{mL}$ of prostaglandin $\mathrm{E}_{1}\left(\mathrm{PGE}_{1}\right.$; Cayman Chemical Co., Ann Arbor, MI, USA) for $10 \mathrm{~min}$, and centrifuged again at $1065 \times g$ for $5 \mathrm{~min}$ (hard spin) to collect resting platelet pellets. Next, platelets were suspended in an appropriate volume of phosphate-buffered saline (PBS) or resuspended in the remaining supernatant (i.e., acellular plasma) after reduction of the volume to adjust the platelet concentration between $2.2 \times 10^{5} / \mu \mathrm{L}$ and $2.8 \times 10^{5} / \mu \mathrm{L}$. Platelets and other blood cell counts were measured using a pocH $100 \mathrm{iV}$ automated hematology analyzer (Sysmex, Kobe, Japan). Leukocytes were not included; therefore, the platelet suspension in plasma was designated as "pure-PRP" (P-PRP).

\section{$c p$-Ti plates and platelet inoculation}

$c p$-Ti plates (Nilaco, Tokyo, Japan) were cut into small, square $10 \times 10 \mathrm{~mm}^{2}$ pieces; washed serially with acetone $(60 \mathrm{~s})$, ethanol $(2 \times 60 \mathrm{~s})$, and distilled water $(2 \times 60 \mathrm{~s})$ in an ultrasonic cleaner (Citizen, Tokyo, Japan), and air-dried.

Platelet suspensions, prepared as described earlier, were inoculated onto the $c p$-Ti plates, and the plates were incubated at ambient temperature for up to $60 \mathrm{~min}$. The $c p$-Ti plates were then vigorously washed two times with PBS on a shaker $(\sim 10 \mathrm{~s})$ and subjected to spectrophotometric assay without fixation or with $10 \%$ neutralized formalin fixation for staining. For activation, platelets in PBS suspensions were treated with $0.1 \% \mathrm{CaCl}_{2}$, as described in previous studies $[11,12]$.

\section{Quantitative and qualitative determination of adherent platelet counts}

Platelets were inoculated onto the surface of $c p$-Ti plates and incubated for $30 \mathrm{~min}$. Then, the $c p$-Ti plates were washed with PBS to remove nonadhered platelets, as described earlier, and were further incubated for $2 \mathrm{~h}$ with a highly water-soluble tetrazolium dye (Cell Counting Kit-8; Dojindo, Kumamoto, Japan). After incubation, $100 \mu \mathrm{L}$ of supernatant was collected, and its absorbance was measured at $450 \mathrm{~nm}$.

Alternatively, platelets were fixed with $10 \%$ neutralized formalin, microperforated with $0.1 \%$ Tween-20-containing PBS (T-PBS) for $1 \mathrm{~min}$, and stained with phalloidin (Cytopainter Phalloidin-iFlour 555 Reagent; Abcam, Cambridge, MA, USA) at ambient temperature in the dark and observed under a fluorescence microscope (ECLIPSE 80i; Nikon, Tokyo, Japan) connected with a cooled CCD camera (VB-7000; Keyence, Osaka, Japan).

\section{Immunocytochemical fluorescence staining}

The $c p$-Ti plates were washed with PBS, and fixed platelets were microperforated with T-PBS for $1 \mathrm{~min}$. The samples were washed twice with PBS and blocked with 0.1\% Block ACE (Sumitomo Dainippon Pharma Co., Ltd., Osaka, Japan) in T-PBS for $1 \mathrm{~h}$. Then, the samples were treated with mouse monoclonal anti-CD62P or anti-CD63 antibody (1:20 dilution; BioLegend, San Diego, CA, USA) for $60 \mathrm{~min}$ at ambient temperature; with rabbit polyclonal anti-fibrinogen (1:16 dilution; MBL, Nagoya, Japan), anti-fibronectin (1:200 dilution; Abcam), anti-vitronectin (1:100 dilution; Abcam), and anti-von Willebrand factor (1:200 dilution; Abcam); or with rabbit monoclonal anti-serum albumin (1:500 dilution; Abcam) overnight at $4{ }^{\circ} \mathrm{C}$. Posttreatment, the samples were again washed twice with T-PBS and then probed with a secondary antibody (i.e., goat anti-mouse IgG H\&L or anti-rabbit IgG H\&L antibody conjugated with Alexa Fluor 488; Abcam) for $60 \mathrm{~min}$ along with phalloidin at ambient temperature in the dark. 
Isotype controls for rabbit primary antibodies (Life Technologies Corporation, Carlsbad, CA, USA) and mouse primary antibodies (Abcam) were used as a negative control.

Finally, after a subsequent wash with PBS, the samples were mounted using an antifade mounting medium (Vectashield; Vector Laboratories, Burlingame, CA, USA), and target proteins were examined under a fluorescence microscope (ECLIPSE 80i; Nikon) connected with a cooled CCD camera (VB-7000; Keyence) [12].

\section{Sample preparation and AFM measurements}

The $c p$-Ti plates with fixed platelets were washed three times with PBS and subjected to atomic force microscope (AFM), as described in previous studies [11, 15]. AFM height images of the samples were recorded in alternating current $(\mathrm{AC})$ mode at room temperature in PBS using the NanoWizard 3 (JPK Instruments AG, Berlin, Germany)
AFM system. Soft cantilevers (Tap300-G; BudgetSensors, Sofia, Bulgaria) were used for scanning.

\section{Statistical analysis}

Data were expressed as mean \pm standard deviation (SD). For two-group comparisons, Student's $t$ test was used to compare mean values (SigmaPlot 12.5; Systat Software, Inc., San Jose, CA, USA). $P<0.05$ was considered statistically significant.

\section{Results}

Figure 1 shows the macroscopic and AFM height images of the plain surface of $c p$-Ti plates. The $c p$-Ti plates were not mirror-finished, but they looked hairline-finished macroscopically. AFM revealed the microstructure of the plain surface of the $c p$-Ti plates. At low magnification, the surface appeared to constitute narrow, straight, hairline-like
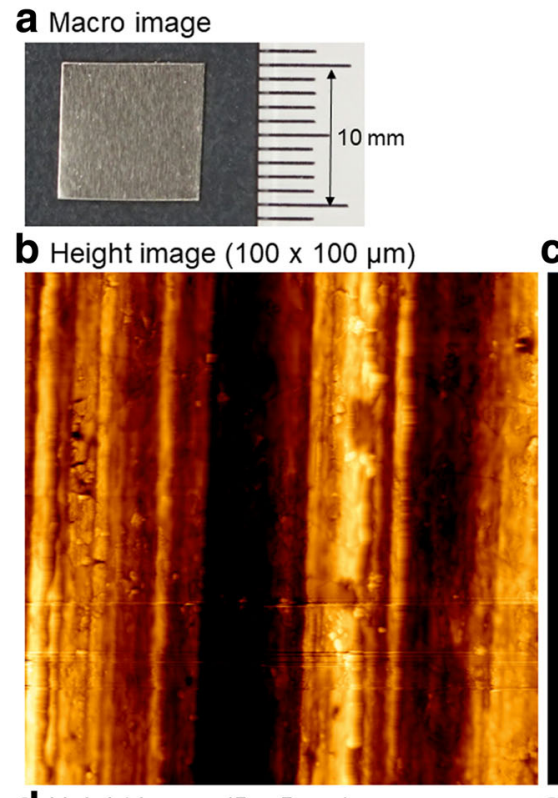

d Height image $(5 \times 5 \mu \mathrm{m})$

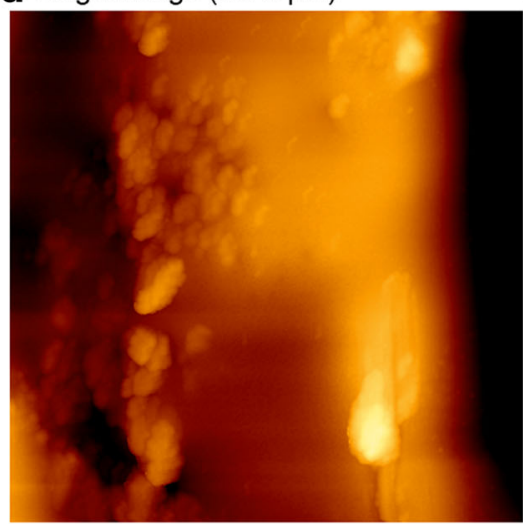

C $3 D$ reconstruction image $(100 \times 100 \mu \mathrm{m})$

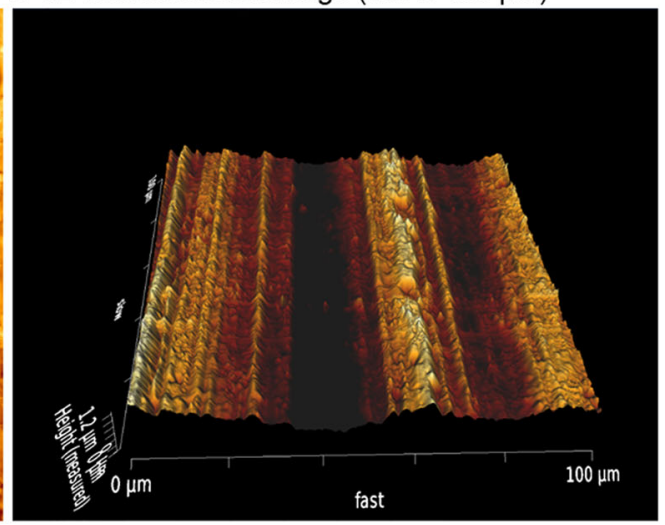

e $3 D$ reconstruction image $(5 \times 5 \mu \mathrm{m})$

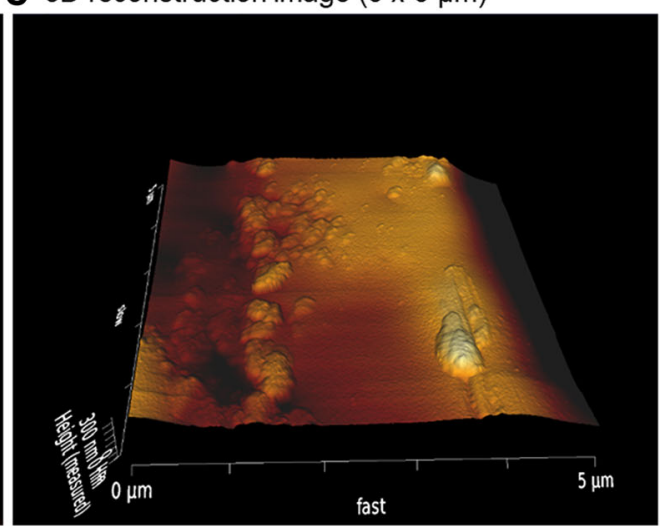

Fig. 1 a Macroscopic and $\mathbf{b}-\mathbf{e}$ AFM images of the plain surface of $c p$-Ti plates. $\mathbf{b}$, d Height images. c, e 3D reconstruction images 
grooves and ridges. However, at high magnification, small, flat spaces were observed between these microstructures.

Figure 2 shows the time-course changes in the number of platelets adhered to the plain surface of $c p$-Ti plates. Platelets in the form of PBS suspensions adhered to the plain surface of the $c p$-Ti plates immediately after inoculation, and the number of adhered platelets increased with time, reaching maximum (i.e., confluence) adhesion at $40 \mathrm{~min}$ of incubation. In contrast, platelets in the form of PRP did not immediately adhere to the plain surface of the $c p$-Ti plates. In addition, although their number increased with time, it did not reach maximum adhesion within $60 \mathrm{~min}$.

Figure 3 shows quantitative evaluations of adhered platelet counts. Platelets incubated onto the plain surface of the $c p$-Ti plates were further treated with a tetrazolium dye, and the intensity of resulting water-soluble formazan dye was spectrophotometrically analyzed. As shown in Fig. 2, platelets in the form of PBS suspensions adhered to the plain surface of the $c p$-Ti plates at a much higher extent compared with those in the form of PRP.

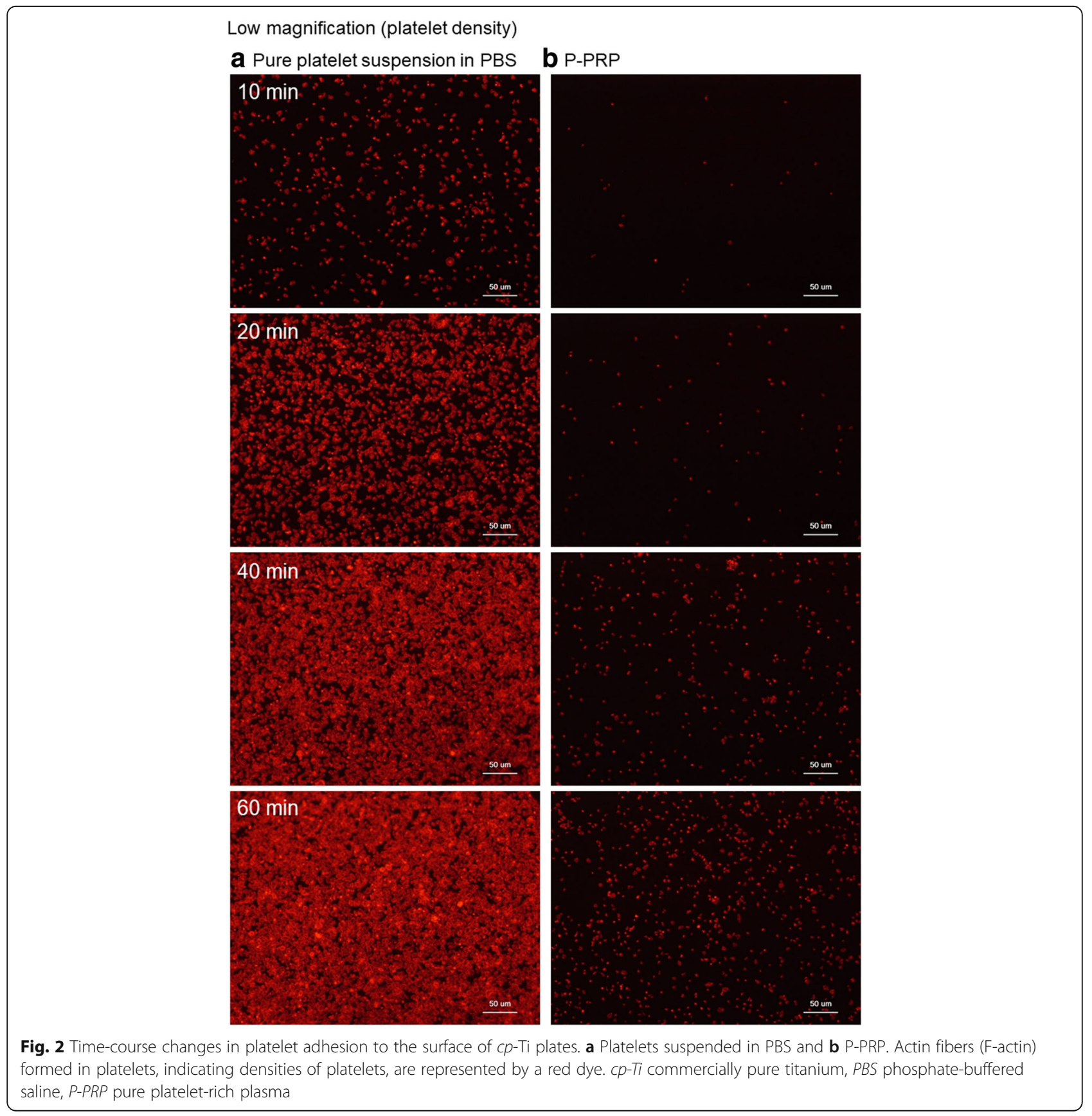




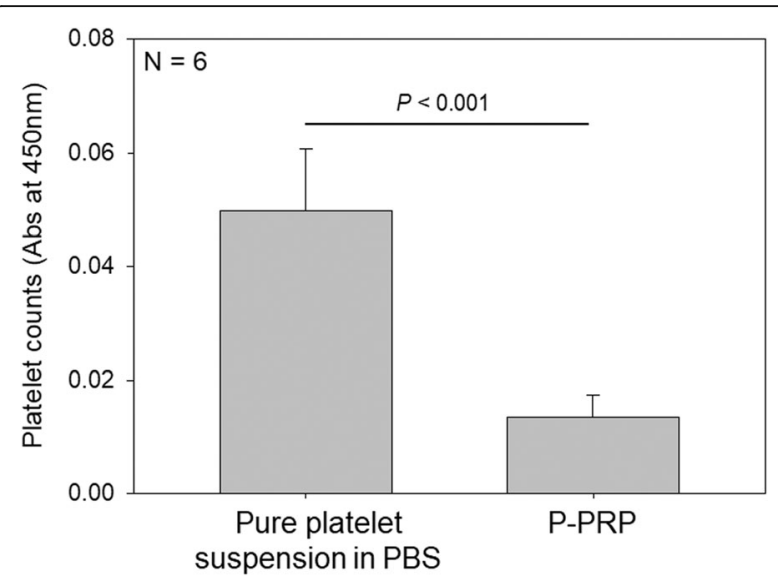

Fig. 3 Quantitative spectrophotometric determination of platelet counts on the surface of $c p$-Ti plates

Figure 4 shows the distribution of FGN on the plain surface of $c p$-Ti plates. In the extraplatelet space, FGN was adsorbed immediately after inoculation, and its levels were maintained for 60 min regardless of the presence or absence of plasma components. In a limited number of platelets, FGN accumulated in the platelet adhesion area, forming spots. Additional file 1: Figure S1A shows the data of negative control using isotype controls (rabbit IgG for anti-FGN, vWF, FN, albumin, and vitronectin [VN]).

Figure 5 depicts the distribution of vWF on the plain surface of $c p$-Ti plates. In the absence of plasma components, vWF immediately accumulated in the platelet adhesion area rather than in the extraplatelet space, and this characteristic distribution was maintained for up to $60 \mathrm{~min}$. Similar findings were obtained in the presence of plasma components. However, compared with FGN, vWF localization in the extraplatelet space seemed disturbed by the presence of plasma components in the initial phase of aggregation, and the number of vWF-positive particles in the extraplatelet space increased with time.

Figure 6 illustrates the distribution of FN on the plain surface of $c p$-Ti plates. FN distribution was somewhat between FGN and the vWF. In the absence of plasma components, FN was distributed almost equally in both the platelet adhesion area and extraplatelet space in the initial phase of aggregation, and it subsequently moved from the extraplatelet space to accumulate in the platelet adhesion area. Notably, in the presence of plasma components, FN was increasingly found both in the platelet adhesion area and extraplatelet space with time.

Figure 7 shows the distribution of serum albumin on the plain surface of $c p$-Ti plates. In the absence of plasma components, serum albumin was rapidly adsorbed onto the plain surface of $c p$-Ti plates. In addition, as the number of adhered platelets increased, initially adsorbed serum albumin was eliminated from the platelet adhesion area, and it gradually decreased in the extraplatelet space. Compared with other platelet adhesion molecules, only a few serum albumin-positive particles were found in the platelet adhesion area. On the contrary to our prediction, in the presence of plasma components, serum albumin was hardly adsorbed onto the plain surface of the $c p$-Ti plates, but it was associated with platelets as if platelets have specific serum albumin-binding sites.

We then examined the distribution of $\mathrm{VN}$ on the plain surface of $c p$-Ti plates (Additional file 1: Figure S2). Despite possible differences in the antibody titers, compared with other platelet adhesion molecules, VN could not be clearly detected in our samples regardless of the presence or absence of plasma components. In general, VN was distributed in the platelet adhesion area rather than in the extraplatelet space.

Furthermore, we examined the effects of adhesion on platelet activity. Figure 8 shows the time-course changes in cytoskeletal actin fiber development in platelets. In the absence of plasma components, sufficient development of cytoskeletal actin fibers was observed throughout the incubation period. In the presence of plasma components, however, the platelet adhesion area was substantially small and cytoskeletal actin fibers were less developed.

Figure 9 shows the expression of CD62P, an activated platelet marker $[16,17]$, in adhered platelets at $20 \mathrm{~min}$ of incubation. In the presence of plasma components, the platelet adhesion area and adhered platelet count were limited and granular localization of CD62P was the lowest, while in the absence of plasma components, granular localization of CD62P was observed. Additional file 1: Figure $\mathrm{S} 1 \mathrm{~B}$ shows the data of negative control using isotype controls (mouse IgG1 and $\mathrm{k}$ for anti-CD62P and CD63).

Figure 10 depicts the expression of the other activated platelet marker CD63 [16, 17] in adhered platelets. As observed with CD62P, granular localization of CD63 was somewhat higher in the absence than in the presence of plasma components.

As a positive control, time-course changes in CD63 expression in adhered platelets activated by $0.1 \% \mathrm{CaCl}_{2}$ were evaluated (Additional file 1: Figure S3). Activated platelets showed upregulated CD62P in the initial phase of aggregation, and this elevated level was maintained for up to $60 \mathrm{~min}$. On the surface of bovine serum albumin (BSA)-coated $c p$-Ti plates, CD63 expression in individual platelets seemed similar to or somewhat less than that on the surface of the control.

\section{Discussion}

Platelet adhesion molecules and dynamic modulation of platelet adhesion

Platelets attach and adhere to the extracellular matrix and endothelial cells or form aggregates with one another 


\section{Fibrinogen (FGN)}

a Pure platelet suspension in PBS
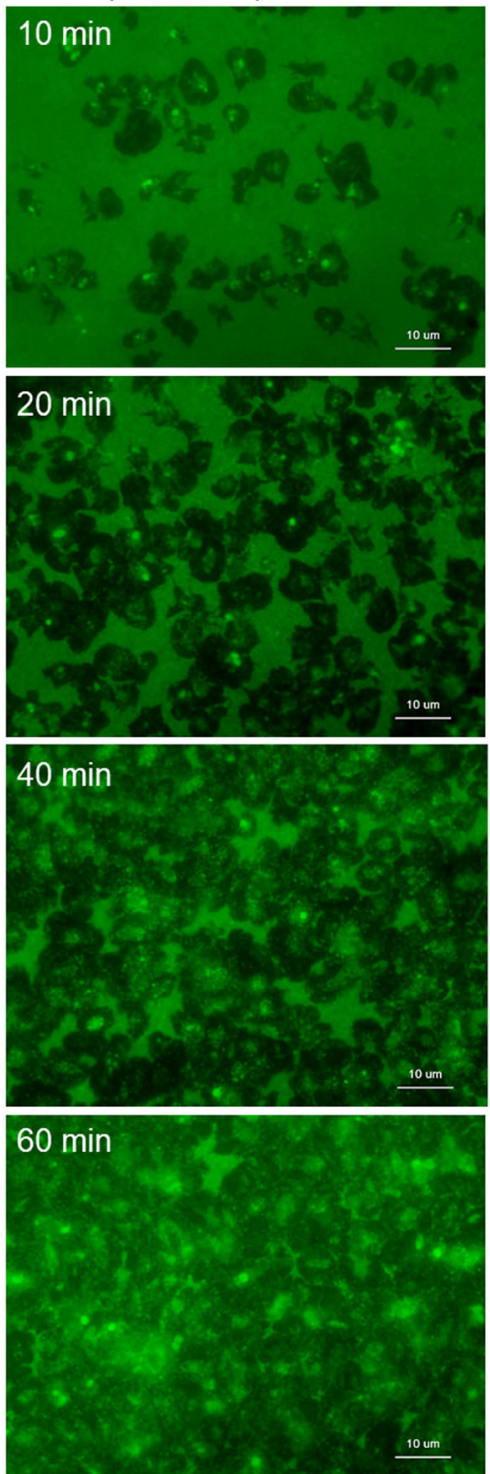

\section{b P-PRP}
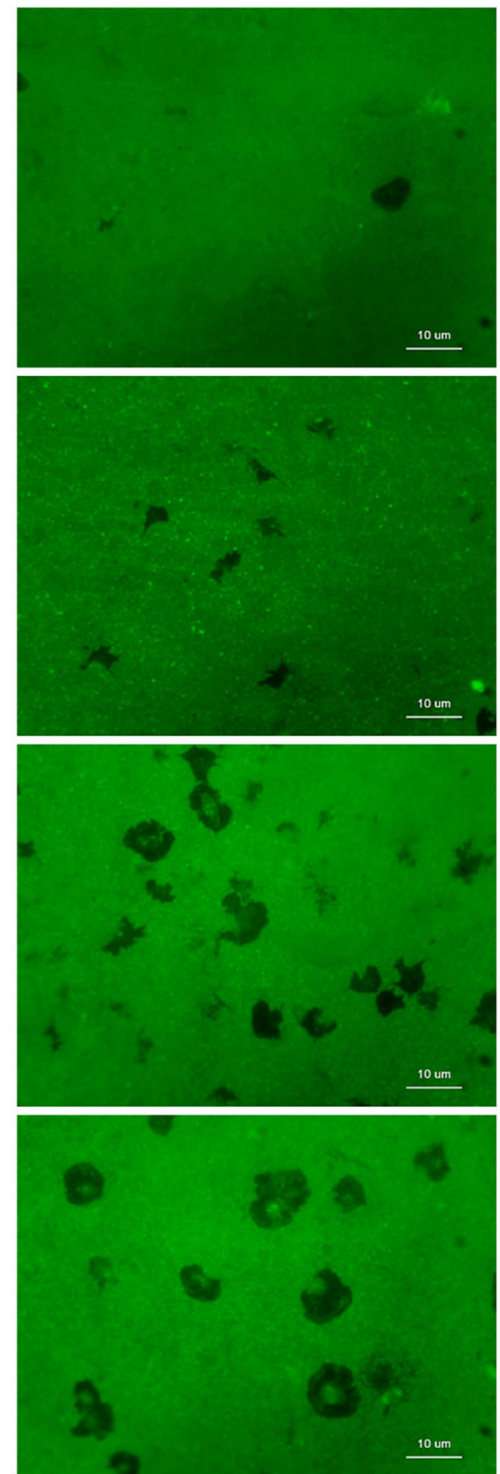

Fig. 4 Distribution of FGN (green dye) on the surface of $c p$-Ti plates. a Platelets suspended in PBS and b P-PRP. FGN fibrinogen, $c p-T i$ commercially pure titanium, PBS phosphate-buffered saline, P-PRP pure platelet-rich plasma

mainly through integrin $\alpha \operatorname{IIb} \beta 3$ (i.e., GPIIbIIIa) and GPIb-V-IX (receptors) during thrombus formation although platelets express various receptors for platelet adhesion molecules [17-19]. In the initial phase of aggregation, resting discoid platelets attach to flat substrates (e.g., vascular endothelial cells in vivo) with filopodia through FGN-integrin $\alpha \operatorname{IIb} \beta 3$ binding [20]. In the subsequent growing phase, in addition to integrin $\alpha \operatorname{IIb} \beta 3$, GPIb-V-IX is involved in aggregation through vWF, FGN, and FN. In the final phase, GPIb-V-IX acts as a predominant adhesion receptor to form rolling aggregates with vWF. These findings indicate that FN and vWF play crucial roles in the initial and late phases of aggregation, respectively.

On the basis of this scenario of in vivo clot formation, possible mechanisms of protein adsorption and platelet adhesion on $c p$-Ti plates are illustrated in Fig. 11. We believe that in the absence of plasma components (unidentified in this study), platelets can be easily activated by contact with $c p$-Ti plates to release adhesion molecules (e.g., FGN, vWF, and FN), which can be predominantly adsorbed onto the surface of $c p$-Ti plates, and adhere to the plates through these adhesion molecules (for more details, see the next subsection). Platelets have 

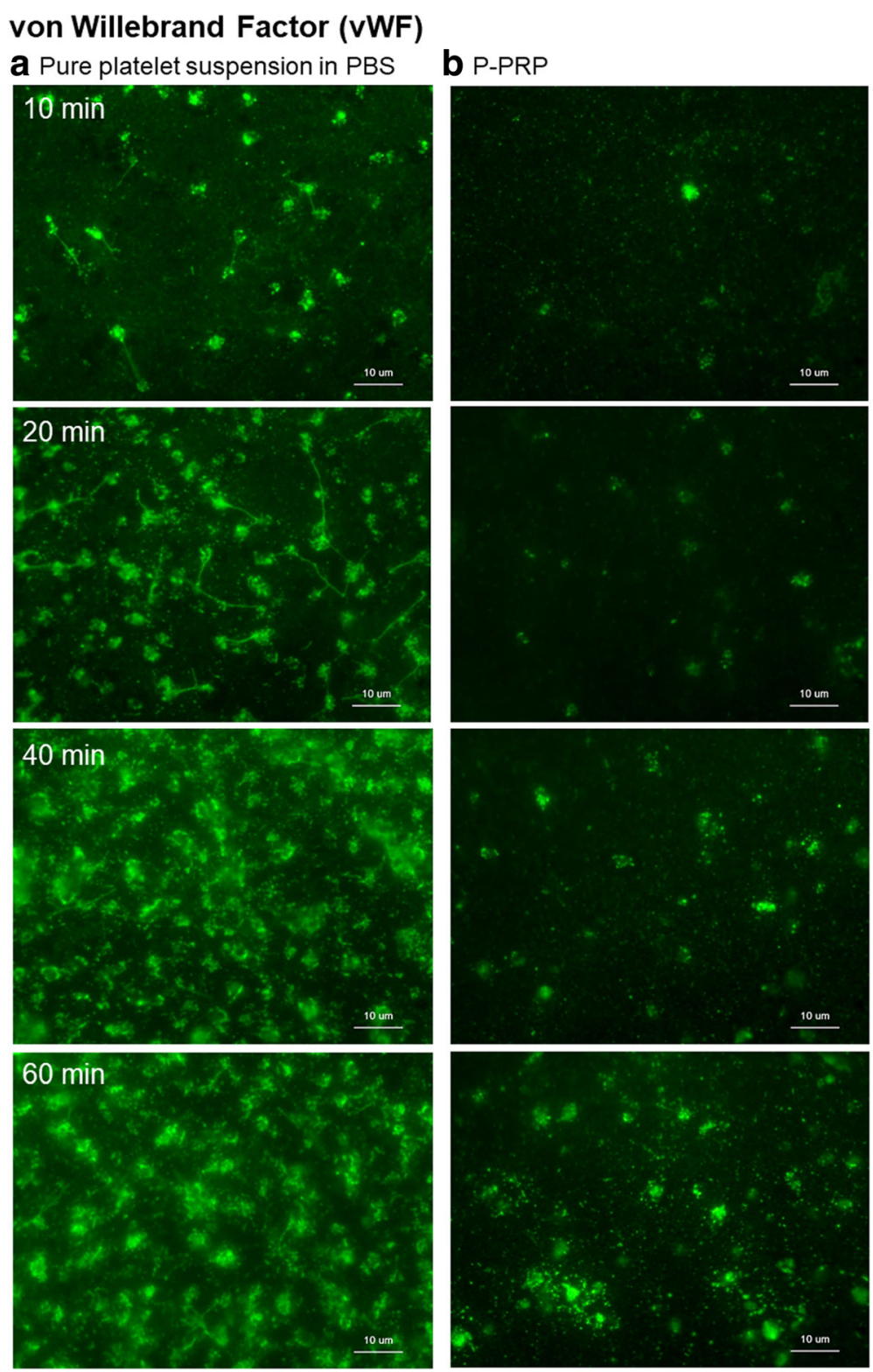

Fig. 5 Distribution of VWF (green dye) on the surface of $c p$-Ti plates. a Platelets suspended in PBS and $\mathbf{b}$ P-PRP. vWF von Willebrand factor, $c p-T i$ commercially pure titanium, PBS phosphate-buffered saline, P-PRP pure platelet-rich plasma

two major types of adhesion systems (i.e., integrin $\alpha \mathrm{IIb} \beta 3$ and GPIb-V-IX) [20]; therefore, in the absence of plasma components, these activated platelets are believed to bind to $c p$-Ti plates more tightly compared to less activated platelets.

\section{Association between plasma components and platelet adhesion molecules}

Platelets accumulate at sites of injury to prevent bleeding and release growth factors to repair damaged tissue and organs in vivo. Under in vitro conditions, a series of these reactions could be modified to some extent against foreign substrates, particularly in the presence of anticoagulants that deplete $\mathrm{Ca}^{2+}$ from coagulation cascades and platelets to prevent platelet activation.

In this study, we observed that platelet adhesion to the plain surface of $c p$-Ti plates was disturbed in the presence of plasma components. Similarly, the adsorption of other platelet adhesion molecules, except FGN, was disturbed in the presence of plasma components. In contrast, in the absence of plasma components, platelets time-dependently adhered to the surface of $c p$-Ti plates 


\section{Fibronectin (FN)}
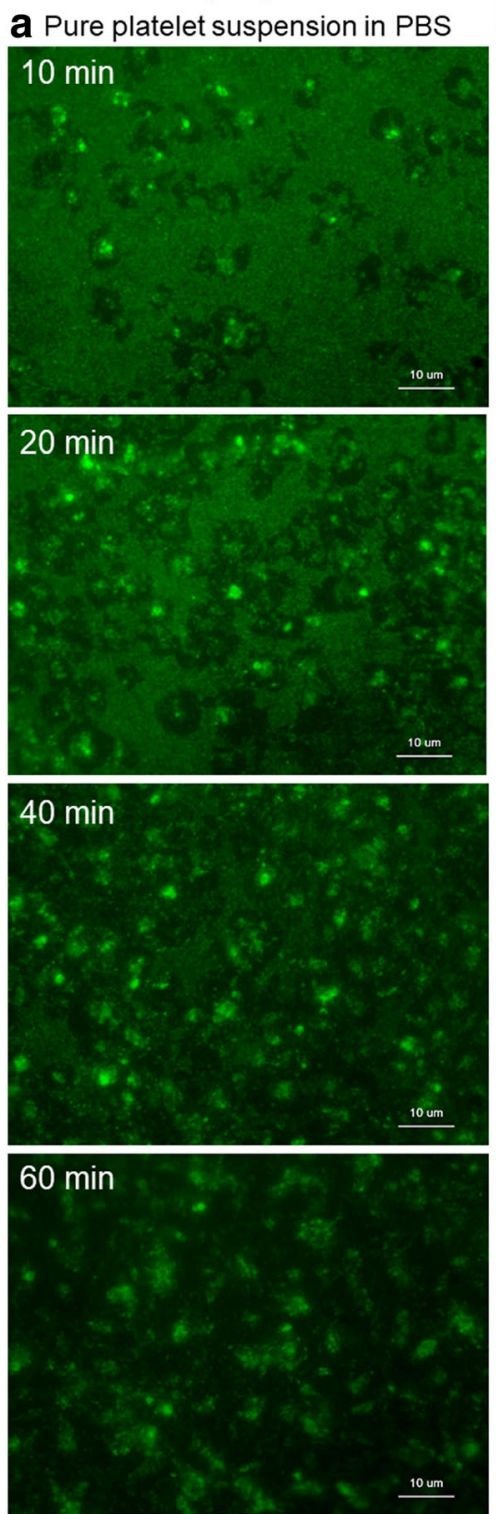

\section{b P-PRP}
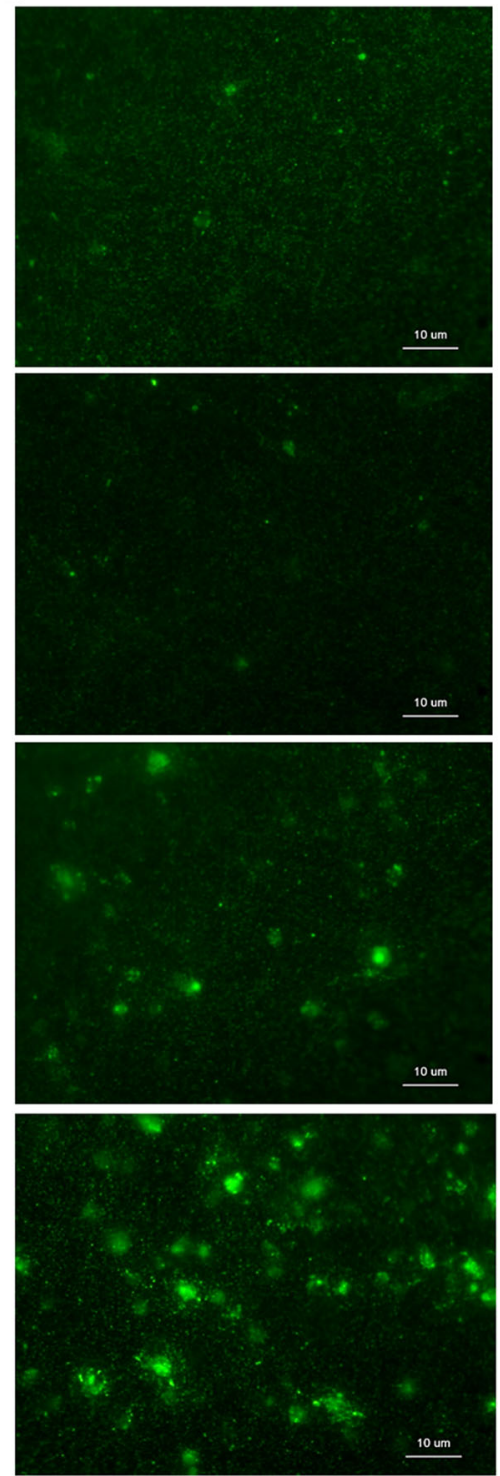

Fig. 6 Distribution of FN (green dye) on the surface of $c p$-Ti plates. a Platelets suspended in PBS and $\mathbf{b}$ P-PRP. FN fibronectin, $c p$-Ti commercially pure titanium, PBS phosphate-buffered saline, P-PRP pure platelet-rich plasma

with well-developed cytoskeletal actin fibers, reaching the maximum adhesion at $40 \mathrm{~min}$ of incubation. In addition, in the absence of plasma components (or, more precisely, in the presence of reduced plasma components), vWF, FGN, and FN were immediately adsorbed on the surface of $c p$-Ti plates.

Taken together, these findings suggest that among platelet adhesion molecules, vWF and FN were favored for platelet adhesion compared with FGN and VN. VN, if present in the platelet suspensions, was not absorbed on $c p$-Ti plate surface or used for adhesion as much as the other platelet adhesion molecules.

\section{Possible mechanisms underlying the blocking action of} serum albumin

In the presence of plasma components, adsorption of platelet adhesion molecules (except FGN) and adhesion of platelets were similarly blocked. Serum albumin is the most abundant plasma protein in humans and several other animals, and BSA [21, 22] is often used as a blocking agent to prevent unspecific protein adsorption onto the surface of devices for handling biological samples [21-23]. However, in this study, although perplexed, albumin was much less visualized on the surface of P-PRP-treated $c p$-Ti plates compared to the control 


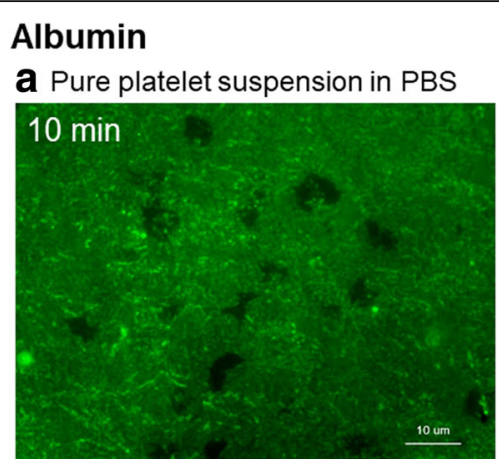

\section{b P-PRP}
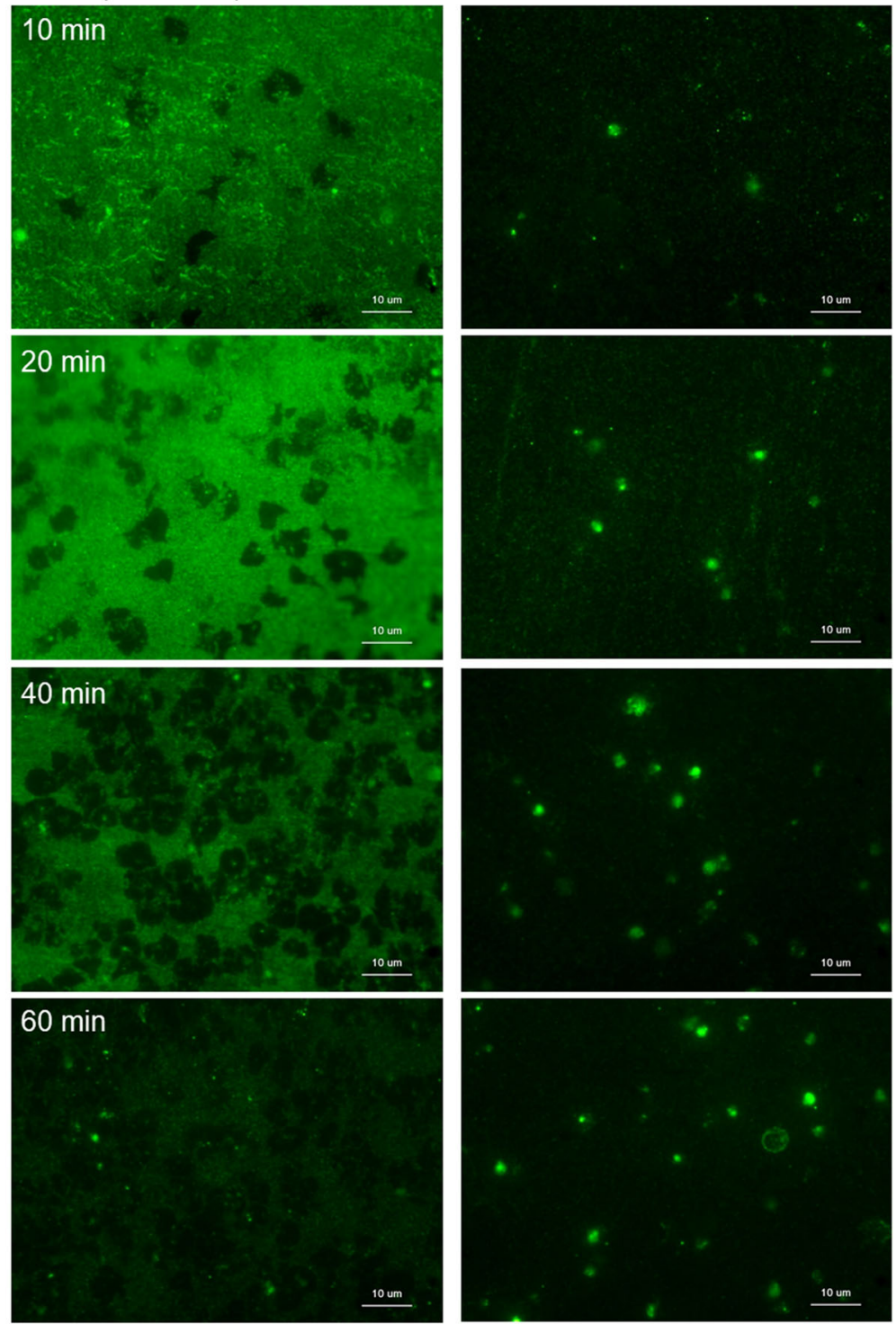

Fig. 7 Distribution of serum albumin (green dye) on the surface of $c p$-Ti plates. a Platelets suspended in PBS and $\mathbf{b}$ P-PRP. $c p$-Ti commercially pure titanium, PBS phosphate-buffered saline, P-PRP pure platelet-rich plasma

surface. This finding suggested two possibilities: (i) serum albumin might not necessarily interfere with platelet adhesion, but, with time, can be replaced gradually with other adhesion molecules, and (ii) serum albumin can be excluded by unidentified plasma components.

Regarding the former possibility, serum albumin lacks any known amino acid sequences or binding protein receptors; therefore, this abundant serum protein cannot support platelet adhesion or aggregation [24, 25]. However, Sivaraman and Lautour [24] proposed that in its adsorption state, serum albumin changes the structure of a large number of charged amino acids (i.e., 24 arginine, 59 lysine, 35 aspartic acid, and 62 glutamic acid residues) recognized by Arg-Gly-Asp (RGD)-specific receptors in platelets similar to an RGD motif. In addition, studies have reported that serum albumin shows significant anticoagulant action in a concentration-dependent manner [26]. According to this theory, serum albumin that remained in platelet pellets produced by centrifugation might be able to support initial platelet adhesion in collaboration with other genuine adhesion molecules. At the very least, it might not be surprising that at low concentrations, serum albumin does not interfere with platelet adhesion under these artificial conditions.

Regarding the latter possibility, we initially hypothesized that PRP-containing serum albumin, whose 


\section{High magnification (development of cytoskeletal actin fibers)}

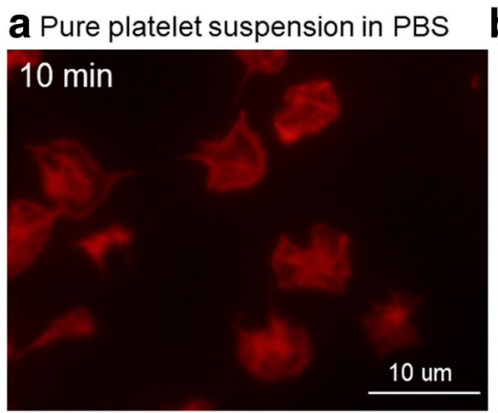

\section{b P-PRP}
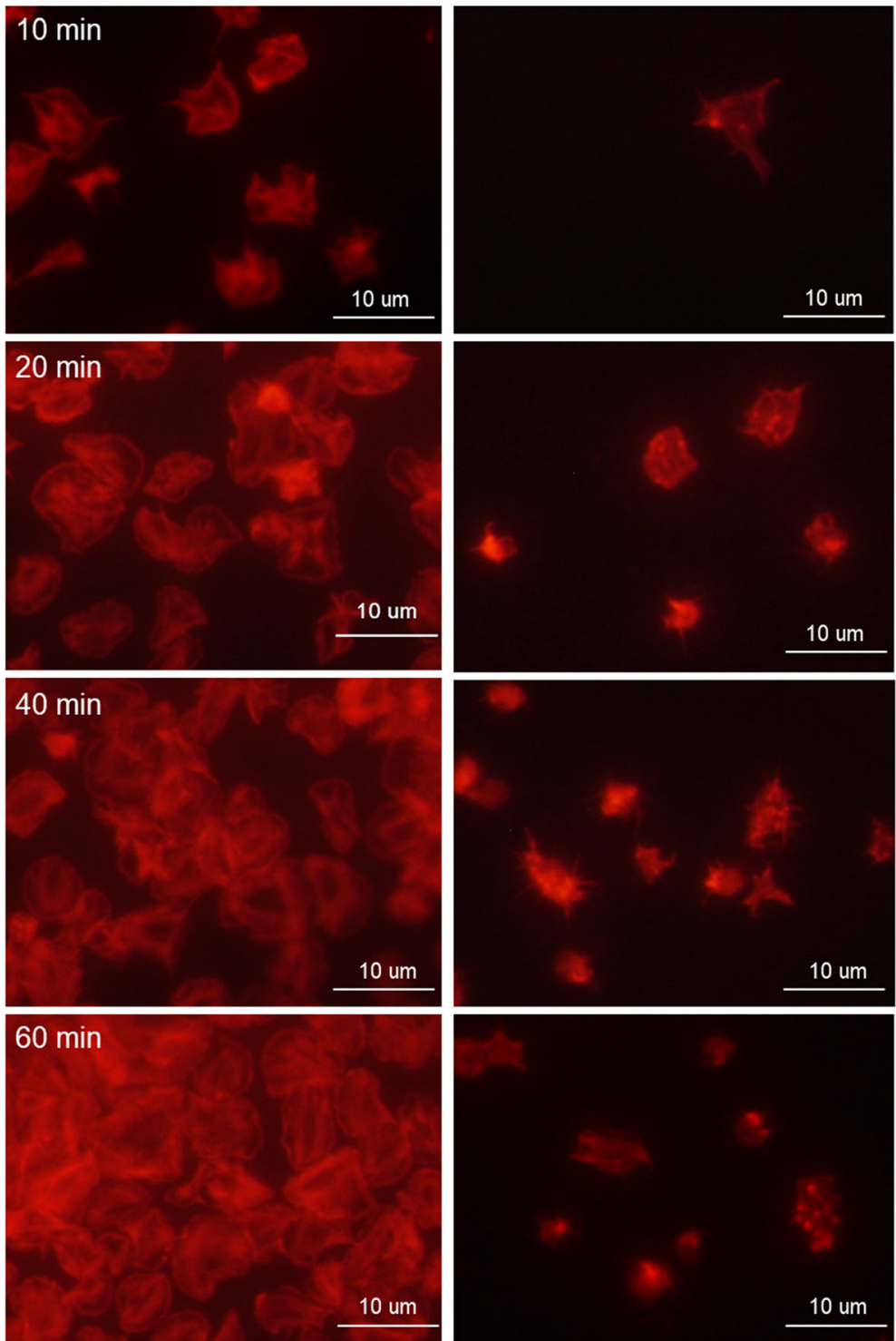

Fig. 8 Time-course of cytoskeletal actin fiber development in adhered platelets at high magnification. a Platelets suspended in PBS and $\mathbf{b}$ P-PRP. PBS phosphate-buffered saline, P-PRP pure platelet-rich plasma

concentration is much higher than in a platelet suspension in PBS, is immediately adsorbed onto the surface of $c p$-Ti plates, blocking platelet adhesion. As expected, in this study, platelet adhesion and vWF and FN adsorption were blocked in the presence of plasma components. However, we did not observe significant levels of albumin adsorbed onto the surface of PRP-treated $c p-\mathrm{Ti}$ plates. This finding suggested that other unidentified plasma components preferentially and rapidly bind to $c p$-Ti plates and reduce the space for albumin adsorption. Otherwise, although albumin is initially adsorbed onto the surface, it can be immediately replaced by other unidentified plasma components. This phenomenon is called the Vroman effect [27]. Although studies have reported that when introduced as the second protein, BSA displaces already existing larger proteins (e.g., FGN and FN) from the surface of $c p$-Ti plates [28]; and in this study, unidentified plasma components like smaller proteins probably displaced the albumin initially adsorbed onto the $c p$-Ti plates. Further studies are required in order to identify these plasma components and elucidate this phenomenon. 


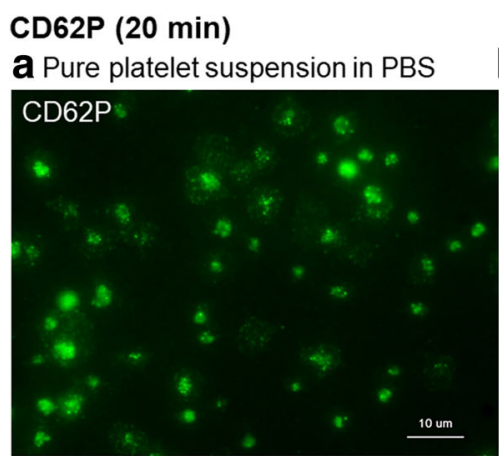

\section{b P-PRP}
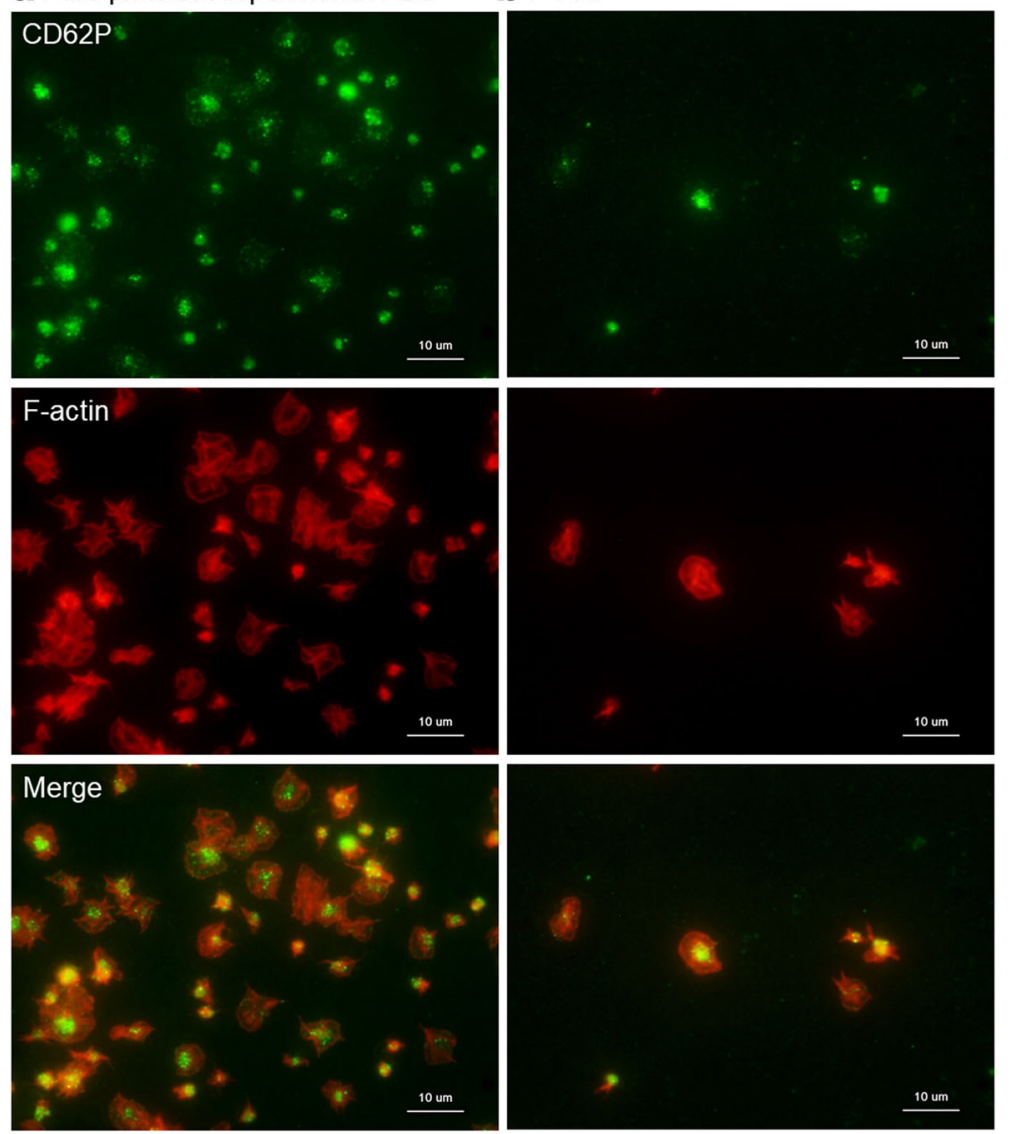

Fig. 9 Expression of CD62P in platelets adhered onto the surface of $c p$-Ti plates. a Platelets suspended in PBS and $\mathbf{b}$ P-PRP. CD62P (green) and F-actin (red) were individually visualized and merged in the bottom panels. $c p$-Ti commercially pure titanium, PBS phosphate-buffered saline, P-PRP pure platelet-rich plasma

\section{Exceptional adsorption of FGN in the presence of plasma components}

Why adsorption of FGN, but not the other adhesion molecules, was not inhibited by plasma components, although the $c p$-Ti surface was not covered by albumin remains unknown. FGN was not adsorbed on the surface of BSA-coated $c p$-Ti plates (Additional file 1: Figure S4). Interestingly, adsorbed FGN (and vWF) can promote platelet adhesion more potently than FN [29], and it is adsorbed on the top of serum albumin in a bilayer or multilayer [23, 28]. Moreover, FGN stimulates platelet adhesion after being adsorbed onto a surface but not in its native form (nonadsorbed) [30], and it associates with $\mathrm{TiO}_{2}$ markedly faster than human serum albumin [31].

In this study, in the absence of plasma components, platelet adhesion molecules were adsorbed together with serum albumin on the surface of $c p$-Ti plates, indicating that these proteins are accumulated on the surface in a multilayer. FGN, but not vWF or FN, in the upper layer may reduce its ability to adhere to platelets.

\section{Platelet activation}

Integrins function as dynamic scaffolds, which facilitate platelet adhesion onto surrounding cells or artificial surfaces, and as receptors for external signaling molecules, such as FGN, vWF, and FN [19, 24, 30, 32-36]. In this study, we examined platelet activation by the development of cytoskeletal actin fibers and the expression of surface markers. Regardless of the number of adhered platelets, F-actin formation was well developed in adhered platelets from earlier phases in the absence of plasma components but poorly developed or absent in the presence of plasma components. Similarly, both CD62P and CD63 were upregulated in a time-dependent manner in the absence of plasma components. These results suggest that plasma components interfere with the adhesion of floating platelets and subsequent activation of adhered platelets.

\section{Possible biomedical significance and clinical relevance}

Finally, the biomedical significance of dental implant pretreatment with P-PRP prior to implantation should 


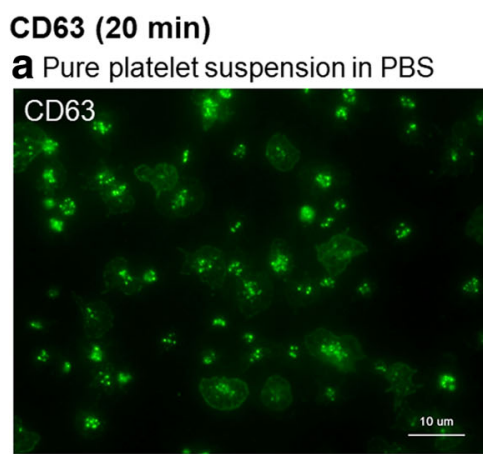

\section{b P-PRP}
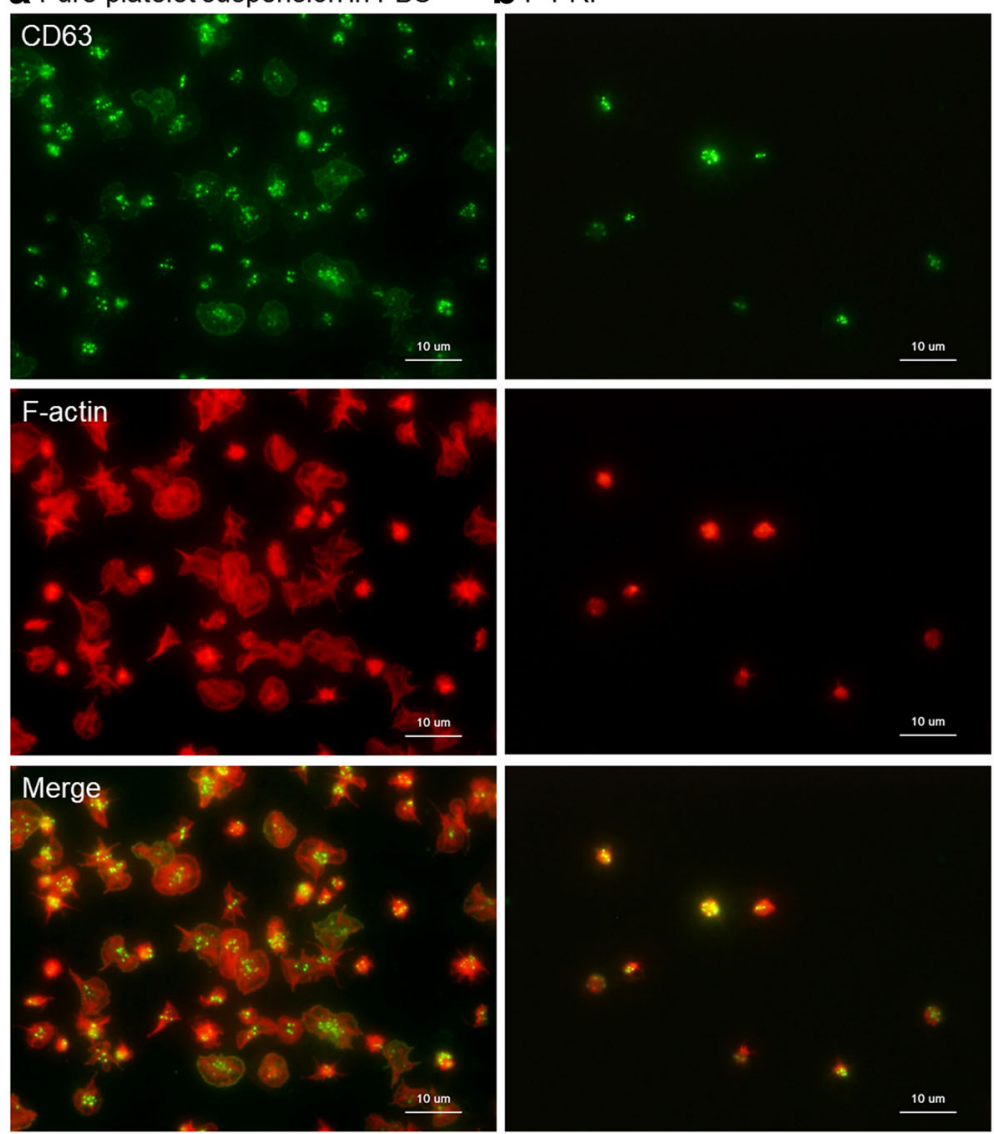

Fig. 10 Expression of CD63 in platelets adhered onto the surface of $c p-T i$ plates. a Platelets suspended in PBS and b P-PRP. CD63 (green) and F-actin (red) were individually visualized and merged in the bottom panels. $c p$-Ti commercially pure titanium, PBS phosphate-buffered saline, P-PRP pure platelet-rich plasma

be briefly discussed. In this study, we observed that the adhesion and adsorption of platelets and major platelet adhesion molecules on the plain surface of $c p$-Ti plates were disturbed in the presence of plasma components. Although we observed that prolonged P-PRP treatment increases platelet adhesion, our findings do not support the theory that rapid pretreatment with P-PRP may positively functionalize the plain surface of $c p$-Ti plates.

Some clinical and preclinical animal studies have supported the use of PRP for better dental implant stability or osseointegration $[3,6,7,10,37]$. However, other studies have reported no positive effects $[4,5$, $8,9]$. Recently developed dental implants have surfaces modified mechanically or (electro) chemically to improve their biocompatibility and tissue integration. The microrough surfaces of dental implants might be suitable to efficiently trap platelets, platelet adhesion molecules, and growth factors such that the dental implant surface is positively functionalized even with rapid pretreatment.
This controversial situation seems identical to that of skeletal regeneration using PRP [38]. PRP is considered an adjuvant therapy, and it largely depends on successful angiogenesis [2,39]. Although growth factors and platelet adhesion molecules can be adsorbed more easily onto the modified surface, e.g., a microrough surface, of dental implants and retained more efficiently, effects which are expected to initially work for better cell adhesion and growth, to our knowledge, there is no convincing evidence that supports better subsequent stabilization and consequent osseointegration.

This lack of evidence might be due to variations in application protocols, differences in bone conditions at the site of implantation, and variations in individual PRP quality. In this study, we examined platelet adhesion under the most popular protocol for PRP applicationcoating by simple immersion. This procedure is superior in terms of complete exclusion of air bubbles generated on the implant surface by visual inspection. To our knowledge, no studies have exclusively performed direct 


\section{a PLT suspended in PBS}

\section{PLT activated by contact with $c p-T i$}
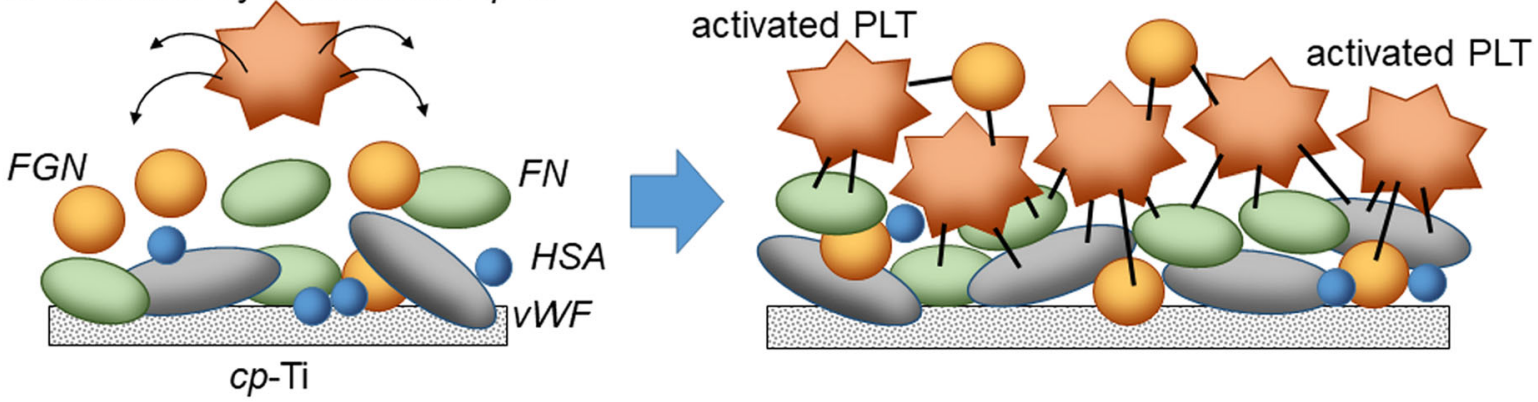

b P-PRP (PLT suspended in plasma)
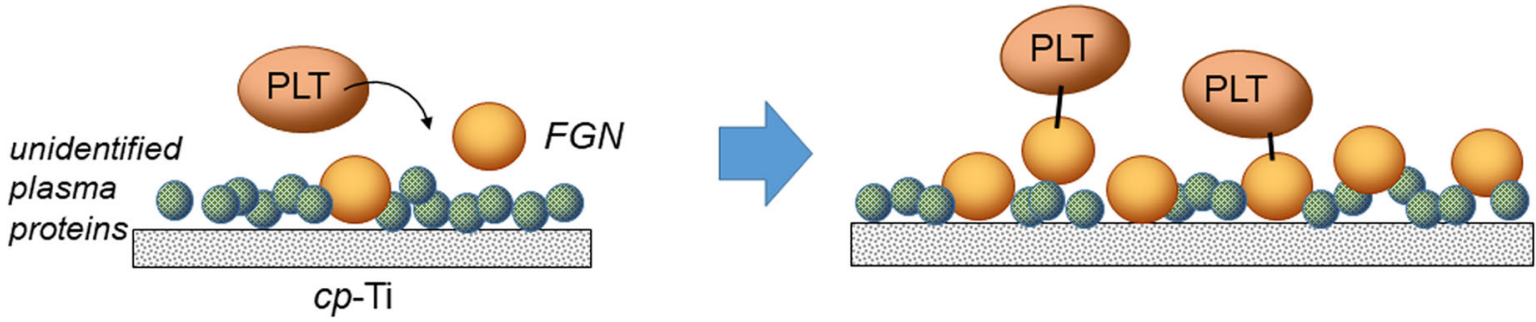

Fig. 11 Possible mechanisms of protein adsorption and platelet adhesion on cp-Ti plates. a Platelets suspended in PBS and $\mathbf{b}$ P-PRP. (Left and center) Time-course changes in protein adsorption and platelet adhesion. (Right) Possible relationships between adhesion molecules and their receptors expressed in platelets. $c P$-Ti commercially pure titanium, PBS phosphate-buffered saline, P-PRP pure platelet-rich plasma, PLT platelet

application of PRP in any form [40] or platelet-rich fibrin (PRF) in implant holes to improve the initial stability of implants. When implant hole conditions are not favorable for mechanically tight contact between bone and implant surface, a clotted PRP or PRF membrane might function like a sealing tape to temporally and mechanically stabilize the implants. However, it is not yet accepted that the functionalization of implants using PRP, like the PRP immersion procedure, contributes to stabilization of placed implants in the medium term.

On the other hand, when bone quality is high, the bone is stiff enough to retain implants tightly and flexibly without the occurrence of a gap during rotation with frictional force. Under these conditions, relatively larger components (e.g., platelets and fibrin) coated on the implant surface are probably easily removed during rotation. Therefore, PRP might not appreciably contribute to further improvement of implant stability, but might somewhat contribute to the improvement of implant bioaffinity by securing platelet-derived small molecules and excluding air bubbles.

Compared to other factors described earlier, we believe that PRP quality is usually a more crucial factor for better clinical outcomes. As described in previous studies, we recommend that clinical implantologists use high-quality PRP preparations for surface modification of implants as in other regenerative therapies $[41,42]$.

\section{Conclusions}

Platelets mainly use vWF and FN for adhesion onto the surface of $c p$-Ti plates. However, this adhesion is substantially disturbed by the presence of plasma nonadhesion molecules. Although the data obtained in this study are limited, therefore, titanium dental implants with a plain surface, such as machined implants, may not be significantly functionalized by a rapid PRP pretreatment.

\section{Additional file}

Additional file 1: Figure S1. Negative controls for immunofluorescent visualization of FGN, vWF, FN, CD62P, and CD63. Control platelets

incubated on $c p$-Ti plates for 20 mins were used. FGN, fibrinogen; VWF, von Willebrand factor; FN, fibronectin; $c p$-Ti, commercially pure titanium. Figure S2. Negative controls for immunofluorescent visualization of FGN, VWF, FN, CD62P, and CD63. Control platelets incubated on cp-Ti plates for 20 mins were used. FGN, fibrinogen; VWF, von Willebrand factor; FN fibronectin; $c p$-Ti, commercially pure titanium. Figure S3. Time-course changes in adhesion of $\mathrm{CD}^{+} 3^{+}$platelets. (A) Platelets suspended in PBS without activation and (B) $0.1 \% \mathrm{CaCl}$-activated platelets suspended in PBS. (Left bottom) Control platelets incubated on BSA-coated $c p$-Ti plates for 20 mins. PBS, phosphate-buffered saline; BSA, bovine serum albumin; 
cp-Ti, commercially pure titanium. Figure 54 . Adsorption of FGN, vWF, FN, and VN onto the surface of BSA-coated $c p$-Ti plates. Platelets suspended in PBS on the (A) control surface and (B) BSA-coated surface. FGN, fibrinogen; VWF, von Willebrand factor; FN, fibronectin; VN, vitronectin; cp-Ti, commercially pure titanium; PBS, phosphate-buffered saline; BSA, bovine serum albumin. (DOCX $2495 \mathrm{~kb})$

\section{Abbreviations}

ACD-A: A formulation of acid-citrate-dextrose solution (new line) AC Alternating current; AFM: Atomic force microscope; BSA: Bovine serum albumin (new line) $c p$-Ti commercially pure titanium; FGN: Fibrinogen; FN: Fibronectin; HSA: Human serum albumin; PGE $_{1}$ : Prostaglandin $E_{1} ;$ PRP: Platelet-rich plasma (new line) P-PRP pure PRP; SD: Standard deviation; T-PBS: 0.1\% Tween-20-containing PBS; VN: Vitronectin; VWF: von Willebrand factor

\section{Acknowledgements}

Not applicable.

\section{Funding}

This study was financially supported in part by JSPS KAKENHI (Grant Number 18 K09595).

\section{Availability of data and materials}

The image data are available from the corresponding author on reasonable request.

\section{Authors' contributions}

AT, ST, TT, and TK conceived and designed the study, performed the experiments and data analysis, and wrote the manuscript. KI, Taisuke W, and Takao W designed and performed the experiments, data analysis, and interpretation. KN conceived the study and participated in discussion of the results and manuscript preparation. All authors read and approved the final version of the manuscript.

\section{Ethics approval and consent to participate}

The study design and consent forms of all the procedures performed were approved by the ethics committee for human participants of the Niigata University School of Medicine (Niigata, Japan) in accordance with the Helsinki Declaration of 1964 as revised in 2013. Written informed consents to participate in the study were obtained from all the participants.

\section{Consent for publication}

Written informed consent for the publication of these were obtained from all the participants.

\section{Competing interests}

Akira Takahashi, Shotaro Takahashi, Tetsuhiro Tsujino, Kazushige Isobe, Taisuke Watanabe, Yutaka Kitamura, Takao Watanabe, Koh Nakata, and Tomoyuki Kawase declare that they have no competing interests.

\section{Publisher's Note}

Springer Nature remains neutral with regard to jurisdictional claims in published maps and institutional affiliations.

\footnotetext{
Author details

${ }^{1}$ Kawasaki, Japan. ${ }^{2}$ Tokyo Plastic Dental Society, Kita-ku, Tokyo, Japan. ${ }^{3}$ Division of Anatomy and Cell Biology of the Hard Tissue, Institute of Medicine and Dentistry, Niigata University, Niigata, Japan. ${ }^{4}$ Department of Oral and Maxillofacial Surgery, Matsumoto Dental University, Shiojiri, Japan. ${ }^{5}$ Department of Oral Science, Graduate School of Dentistry, Kanagawa Dental University, Yokosuka, Japan. ${ }^{6}$ Bioscience Medical Research Center, Niigata University Medical and Dental Hospital, Niigata, Japan. ${ }^{7}$ Division of Oral Bioengineering, Institute of Medicine and Dentistry, Niigata University, Niigata, Japan.
}

Received: 20 September 2018 Accepted: 14 January 2019

Published online: 25 February 2019

\section{References}

1. Golebiewska EM, Poole AW. Platelet secretion: from haemostasis to wound healing and beyond. Blood Rev. 2015;29:153-62.

2. Kawase T. Platelet-rich plasma and its derivatives as promising bioactive materials for regenerative medicine: basic principles and concepts underlying recent advances. Odontology. 2015;103:126-35.

3. Anitua E, Orive G, Pla R, Roman P, Serrano V, Andia I. The effects of PRGF on bone regeneration and on titanium implant osseointegration in goats: a histologic and histomorphometric study. J Biomed Mater Res A. 2009;91:158-65.

4. Ergun G, Egilmez F, Cekic-Nagas I, Karaca IR, Bozkaya S. Effect of platelet-rich plasma on the outcome of early loaded dental implants: a 3-year follow-up study. J Oral Implantol. 2013;39:256-63.

5. Garcia RV, Gabrielli MAC, Hochuli-Vieira E, Spolidorio LC, Filho JGP, Neto FAD, LAGd C, Shibli JA. Effect of platelet-rich plasma on peri-implant bone repair: a histologic study in dogs. J Oral Implantol. 2010;36:281-90.

6. Kim SG, Chung CH, Kim YK, Park JC, Lim SC. Use of particulate dentin-plaster of Paris combination with/without platelet-rich plasma in the treatment of bone defects around implants. Int J Oral Maxillofac Implants. 2002;17:86-94.

7. Kundu R, Rathee M. Effect of platelet-rich-plasma (PRP) and implant surface topography on implant stability and bone. J Clin Diagn Res. 2014;8:Zc26-30.

8. Monov G, Fuerst G, Tepper G, Watzak G, Zechner W, Watzek G. The effect of platelet-rich plasma upon implant stability measured by resonance frequency analysis in the lower anterior mandibles. Clin Oral Implants Res. 2005;16:461-5.

9. Streckbein P, Kleis W, Buch RS, Hansen T, Weibrich G. Bone healing with or without platelet-rich plasma around four different dental implant surfaces in beagle dogs. Clin Implant Dent Relat Res. 2014;16:479-86.

10. Zechner W, Tangl S, Tepper G, Furst G, Bernhart T, Haas R, Mailath G, Watzek $\mathrm{G}$. Influence of platelet-rich plasma on osseous healing of dental implants: a histologic and histomorphometric study in minipigs. Int J Oral Maxillofac Implants. 2003;18:15-22.

11. Kitamura Y, Isobe K, Kawabata H, Tsujino T, Watanabe T, Nakamura M, Toyoda T, Okudera H, Okuda K, Nakata K, Kawase T. Quantitative evaluation of morphological changes in activated platelets in vitro using digital holographic microscopy. Micron. 2018;113:1-9.

12. Toyoda T, Isobe K, Tsujino T, Koyata Y, Ohyagi F, Watanabe T, Nakamura M, Kitamura Y, Okudera H, Nakata K, Kawase T. Direct activation of platelets by addition of $\mathrm{CaCl} 2$ leads coagulation of platelet-rich plasma. Int J Implant Dent. 2018:4:23.

13. Isobe K, Suzuki M, Watanabe T, Kitamura Y, Suzuki T, Kawabata H, Nakamura M, Okudera T, Okudera H, Uematsu K, Nakata K, Tanaka T, Kawase T. Plateletrich fibrin prepared from stored whole-blood samples. Int J Implant Dent. 2017;3:6.

14. Kawabata H, Isobe K, Watanabe T, Okudera T, Nakamura M, Suzuki M, Ryu J, Kitamura Y, Okudera H, Okuda K, Nakata K, Kawase T. Quality assessment of platelet-rich fibrin-like matrix prepared from whole blood samples after extended storage. Biomedicine. 2017:5:57.

15. Horimizu M, Kawase T, Nakajima Y, Okuda K, Nagata M, Wolff LF, Yoshie H. An improved freeze-dried PRP-coated biodegradable material suitable for connective tissue regenerative therapy. Cryobiology. 2013;66:223-32.

16. Gupta S, Reviakine I. Platelet activation profiles on TiO2: effect of $\mathrm{Ca} 2+$ binding to the surface. Biointerphases. 2012;7:28.

17. Daugirdas JT, Bernardo AA. Hemodialysis effect on platelet count and function and hemodialysis-associated thrombocytopenia. Kidney Int. 2012; 82:147-57.

18. Ghoshal K, Bhattacharyya M. Overview of platelet physiology: its hemostatic and nonhemostatic role in disease pathogenesis. Sci World J. 2014;2014:781857.

19. Nieswandt B, Varga-Szabo D, Elvers M. Integrins in platelet activation. J Thromb Haemost. 2009;7(Suppl 1):206-9.

20. Jackson SP. The growing complexity of platelet aggregation. Blood. 2007; 109:5087-95.

21. Goodman SL, Cooper SL, Albrecht RM. The effects of substrate-adsorbed albumin on platelet spreading. J Biomater Sci Polym Ed. 1991;2:147-59.

22. Maalej N, Albrecht R, Loscalzo J, Folts JD. The potent platelet inhibitory effects of S-nitrosated albumin coating of artificial surfaces. J Am Coll Cardiol. 1999;33:1408-14

23. Holmberg M, Hou X. Fibrinogen adsorption on blocked surface of albumin. Colloids Surf B: Biointerfaces. 2011;84:71-5. 
24. Sivaraman B, Latour RA. The relationship between platelet adhesion on surfaces and the structure versus the amount of adsorbed fibrinogen. Biomaterials. 2010;31:832-9.

25. Mikhailidis DP, Barradas MA, Maris A, Jeremy JY, Dandona P. Fibrinogen mediated activation of platelet aggregation and thromboxane $A 2$ release: pathological implications in vascular disease. J Clin Pathol. 1985;38:1166-71.

26. Paar M, Rossmann C, Nusshold C, Wagner T, Schlagenhauf A, Leschnik B, Oettl K, Koestenberger M, Cvirn G, Hallstrom S. Anticoagulant action of low, physiologic, and high albumin levels in whole blood. PLoS One. 2017;12: e0182997.

27. Hirsh SL, McKenzie DR, Nosworthy NJ, Denman JA, Sezerman OU, Bilek MM. The Vroman effect: competitive protein exchange with dynamic multilayer protein aggregates. Colloids Surf B: Biointerfaces. 2013;103:395-404.

28. Pegueroles M, Tonda-Turo C, Planell JA, Gil FJ, Aparicio C. Adsorption of fibronectin, fibrinogen, and albumin on $\mathrm{TiO} 2$ : time-resolved kinetics, structural changes, and competition study. Biointerphases. 2012;7:48.

29. Grunkemeier JM, Tsai WB, McFarland CD, Horbett TA. The effect of adsorbed fibrinogen, fibronectin, von Willebrand factor and vitronectin on the procoagulant state of adherent platelets. Biomaterials. 2000;21:2243-52.

30. Childers EP, Peterson Gl, Ellenberger AB, Domino K, Seifert GV, Becker ML. Adhesion of blood plasma proteins and platelet-rich plasma on I-valinebased poly(ester urea). Biomacromolecules. 2016;17:3396-403.

31. Canoa P, Simon-Vazquez R, Popplewell J, Gonzalez-Fernandez A. A quantitative binding study of fibrinogen and human serum albumin to metal oxide nanoparticles by surface plasmon resonance. Biosens Bioelectron. 2015;74:376-83.

32. Shattil SJ, Newman PJ. Integrins: dynamic scaffolds for adhesion and signaling in platelets. Blood. 2004;104:1606-15.

33. Andrews RK, Lopez JA, Berndt MC. Molecular mechanisms of platelet adhesion and activation. Int J Biochem Cell Biol. 1997;29:91-105.

34. Bryckaert M, Rosa J-P, Denis CV, Lenting PJ. Of von Willebrand factor and platelets. Cell Mol Life Sci. 2015;72:307-26.

35. Corum LE, Hlady $V$. The effect of upstream platelet-fibrinogen interactions on downstream adhesion and activation. Biomaterials. 2012;33:1255-60.

36. Watson SP. Platelet activation by extracellular matrix proteins in haemostasis and thrombosis. Curr Pharm Des. 2009;15:1358-72.

37. Georgakopoulos I, Tsantis S, Georgakopoulos P, Korfiatis P, Fanti E, Martelli M, Costaridou L, Petsas T, Panayiotakis G, Martelli FS. The impact of platelet rich plasma (PRP) in osseointegration of oral implants in dental panoramic radiography: texture based evaluation. Clin Cases Miner Bone Metab. 2014; 11:59-66.

38. Roffi A, Filardo G, Kon E, Marcacci M. Does PRP enhance bone integration with grafts, graft substitutes, or implants? A systematic review. BMC Musculoskelet Disord. 2013;14:330.

39. Martínez CE, Smith PC, Palma Alvarado VA. The influence of platelet-derived products on angiogenesis and tissue repair: a concise update. Front Physiol. 2015;6:290.

40. Kawase T, Tanaka T. An updated proposal for terminology and classification of platelet-rich fibrin. Regen Ther. 2018 in press

41. Kawase T, Okuda K. Comprehensive quality control of the regenerative therapy using platelet concentrates: the current situation and prospects in Japan. Biomed Res Int. 2018;2018:10.

42. Kawase T, Takahashi A, Watanabe T, Tsujino T. Proposal for point-of care testing of platelet-rich plasma. Int J Growth Factor Stem Cells Dent. 2019 in press

\section{Submit your manuscript to a SpringerOpen ${ }^{\circ}$ journal and benefit from:}

- Convenient online submission

- Rigorous peer review

- Open access: articles freely available online

- High visibility within the field

- Retaining the copyright to your article

Submit your next manuscript at $\boldsymbol{\nabla}$ springeropen.com 